\title{
Intra-abdominal undescended testis associated with seminoma manifesting as acute appendicitis; An extremely rare clinical presentation; Case report
}

\author{
Leila Ghafoor and Abbas Hajian* \\ Department of general surgery, Kashan University of Medical Sciences, Iran \\ *Corresponding author: Abbas Hajian, Medical doctor, General surgeon, Department of general surgery, Kashan University of Med- \\ ical Sciences, Kashan, Iran.
}

To Cite This Article: Leila Ghafoor and Abbas Hajian, Intra-Abdominal Undescended Testis Associated with Smanifesting as Acute Appendicitis; An Extremely Rare Clinical Presentation; Case Report. Am J Biomed Sci \& Res. 2021 - 12(4). AJBSR.MS.ID.001764.

DOI: 10.34297/AJBSR.2021.12.001764.

Received: 钢 April 04, 2020; Published: 眥 April 14, 2021

\begin{abstract}
Background: Cryptorchidism is a disease almost seen among premature male neonate. It should be underwent surgical fixation to scrotal sac if spontaneous testicular descending was not occurred during the first year of the life. Intra-abdominal undescended testis is a rare condition which makes the testicle susceptible to malignant degeneration. Acute appendicitis has varied presentation according to variety of appendix location. Manifestation of undescended intra-abdominal testis associated with seminoma degeneration as an acute appendicitis is an extremely rare clinical presentation.
\end{abstract}

Case presentation: We introduced in this report a 39 years old man who was the second case of the literature that underwent emergent open appendectomy with underlying intra-abdominal undescended testis associated with seminoma which completely resected following appendectomy.

Conclusion: An undescended intra-abdominal right testis could manifest itself primarily as acute appendicitis.

Abbreviation: GCT: Germ Cell Tumor; UDT: Undescended Testis

\section{Introduction}

Primary cryptorchidism is an unusual condition with prevalence of $3 \%$ among newborn term boys, although it is seen in $30 \%$ of premature male infants [1]. From all undescended testes (UDT) most of them are palpable in inguinal canal or just prior to scrotal space, however, still up to $20 \%$ of UDTs are non-palpable and among the latter about $50-60 \%$ are located in abdominal cavity [2]. Because most of UDTs spontaneously move and fill the empty scrotum during the first year of life, surgical orchidopexy is reserved for boys with remained UDT after this period [1,2]. Untreated UDT particularly intra-abdominal type is susceptible for malignant degeneration because of exposure to higher core body temperature [1]. Namely over $50 \%$ of testicular tumors occurred in testes failed to descend [3]. Germ cell tumors (GCT) involve 95\% of all testicular malignancies in which over $50 \%$ are diagnosed as seminoma [1].

Acute appendicitis is the most common surgical disease involves $8 \%$ of all people through their life. Although it is mostly seen in second and third decades of life still all age groups are at risk [2]. According to anatomical positioning of the appendix, variety of clinical signs and symptoms are expected. Based on an experienced surgeon's judge presence of typical history and clinical finding is adequate for the diagnosis of acute appendicitis in a male patient and no extra imaging study is required to schedule emergent operation.

In this report we introduced the second case of intra-abdominal UDT associated with seminoma development in the literature that 
went under operation with diagnosis of acute appendicitis in lined with the SCARE 2018 criteria [4]. The first identical case was presented in 1986 [5].

\section{Case Presentation}

A 39 years old man referred to surgical emergent department with abdominal pain. He suffered from epigastric and preumbilical pain since the morning of the day he sought medical care. The pain classically shifted to the right iliac fossa as known in acute appendicitis. Nausea, vomiting and hyporexia were also present. Pain was reinforced by movement or coughing. He didn't experience any identical abdominal pain previously in his life. He didn't regularly smoke and denied drink alcohol or any psychiatric disorder. He had a controlled hypertension with daily single tablet of lozartan $25 \mathrm{mg}$. Prior surgical history was positive for right sided orchiectomy following unilateral right UDT in addition to left sided varicocelectomy. However, he denied premature born. The latter surgeries were performed when he was in his 29 years in one session. Retrospective review of his medical file revealed that the orchiectomy was completed in doubt; while a fat mass suspicious for hypotrophied undescended testicle had been resected from right inguinal canal by an urologist. Although gross pathologic report contained a gray piece of tissue measuring $5 \times 1 \times 1 \mathrm{~cm}$, microscopic description revealed a fat tissue contained congested vessels and one fibrotic area resembling fibrotic testis, however, testicular tissue was not seen. Additionally postoperative abdominopelvic imaging report after orchiectomy was otherwise normal with no sign of intra-abdominal remained UDT. Patient was married and had a single healthy 8 years old girl. In systematic review patient mentioned low grade fever and hyporexia with no additional weight loss, respiratory symptoms, referral pain, hematuria, dysuria, or constipation in recent course of disease. Physical examination initiated beside the patient's bed while he was laid and tended to not move. Although he was ill, the vital signs were completely normal. Abdominal examination was in lined with the diagnosis of acute appendicitis; means the presence of right iliac fossa point tenderness, rebounding pain, and positive rovsing sign. He was otherwise normal in physical examination. Laboratory data also implied on neutrophilic leukocytosis with absolute neutrophilic count $8.2 \times 109 / \mathrm{L}$. According to patient's history and clinical findings he was prepared for emergent appendectomy with no extra imaging study requirement for diagnosis of acute appendicitis. In operating room, following the general anesthesia induction classic Mcburney's incision on right iliac fossa was made. An assistant professor of general surgery, a general surgery chief resident and a licensed aid with operating room educational degree were present at the operating bed. After opening peritoneal layer slight reactive fluid was drained. The cecum was investigated and later the surgeon faced to appendix with inflammatory changes which was fixed to a retroperitoneal mass in its retrocecal location.
Bundles were adhesiolyzed and primary appendectomy was completed. Then retrocecal mass with diameter of $6-7 \mathrm{~cm}$ was examined. We primarily considered it as right dislocated kidney but intraoperative palpation of right kidney in its expected anatomical location ruled out our consideration. Therefore exploration of the mass from the same incision was proceeded. Figure 1 shows intraoperative view of the mass.

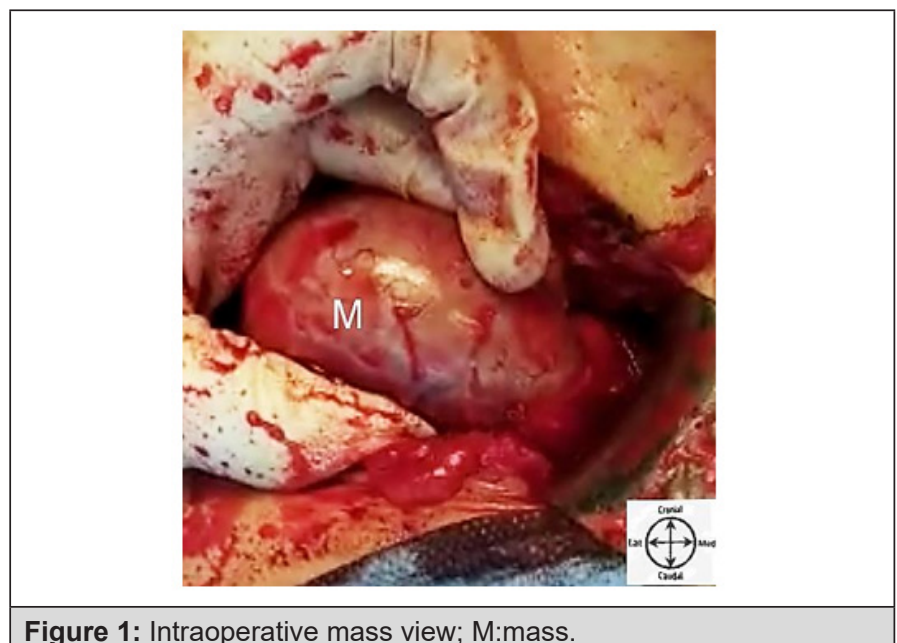

Figure 1: Intraoperative mass view; M:mass.

It was a white oval shaped soft mass with regular burden which contained single process originating from one of its pole remembered enlarged testicle. Figure 2 shows the mentioned objectives. Hence, intraoperative real-time urologic consult was performed through an internet connection with an experienced urologist. He was confirmed our diagnosis of that the mass was a remained intra-abdominal UDT. Foley catheter was introduced and no change was observed in urine characteristic after manipulation of the mass. The remained UDT had no invasions to adjacent structures grossly. Operation was continued with total right sided orchiectomy and finished without complication. Figure 3 shows the totally excised UDT. Pathologic study approved testicular tissue containing unifocal tumoral degeneration in lined with classic type of seminoma which was limited to testis with microscopic lymphovascular invasion around the epididymis.

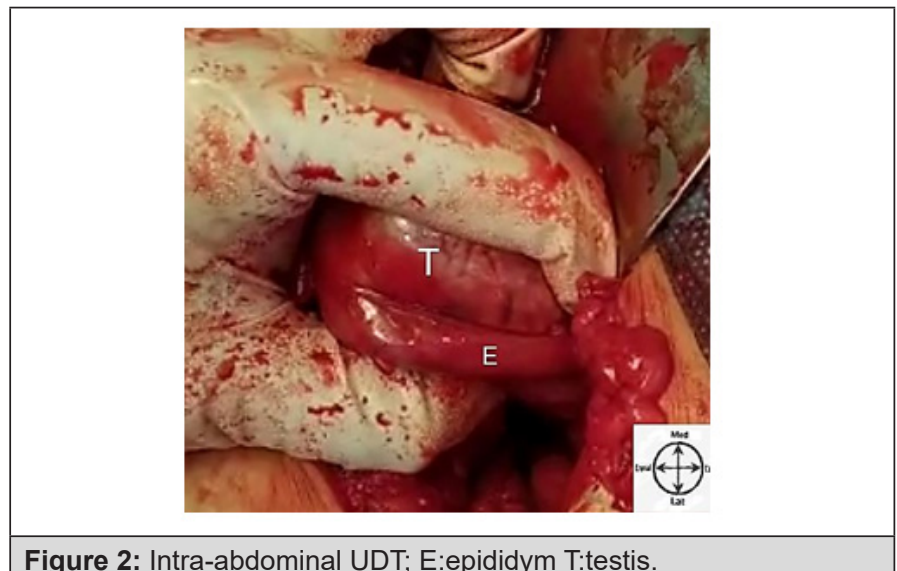




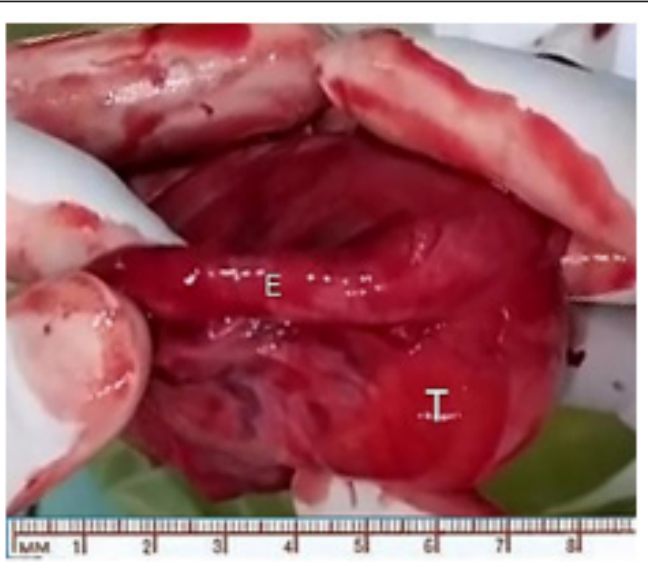

Figure 3: Completely resected UDT; E:epididym T:testis.

Microscopic evaluation of appendix also was concluded in follicular hyperplasia with no sign of tumor invasion. Patient was discharged on the day after surgery and follow up examination after 3 weeks had no abnormal findings. Postoperative metastatic workup was performed by computed tomographic imaging study of lung, abdomen and pelvic which all were intact and free of metastatic lesion. He was referred to an oncologist and underwent adjuvant chemotherapy.

\section{Discussion}

Any painful testicular mass should consider testicular cancer until proven otherwise. Testicular cancer is the most common malignant degeneration in males between 20-40 years. Also among 15-19 years teenagers it is the second most common cancer. In elderly, primary testicular cancer is rare and metastatic lymphoma is mostly observed. Among younger males retroperitoneal lymphadenopathy is equal to testicular cancer till pathologic study on specimen biopsy ruled it out. There are risk factors for testicular cancer which UDT is the leading cause followed by positive family or personal history of testicular cancer, and intratubular germ cell neoplasia [1]. An empty scrotal sac with palpable mass in inguinal canal is highly suggestive for diagnosis of the UDT. If physician could not palpate the testicle intra-abdominal UDT is suspected. However, evidence showed that either non-palpable or intra-abdominal testicle was present among 50-60\% of cases [3]. Transverse testicular ectopia is also a rare condition in which both of testicles locate in ipsilateral scrotal sac and in contralateral side there is either empty scrotum or non-palpable testicle [6]. Secondary testicular dislocation following traumatic pelvic ring injury whether unilateral or bilateral was reported which present like primary UDT with empty scrotal sac and intra-abdominal testicles [7]. Our patient had no previous history of pelvic trauma. Cryptorchidism is most commonly seen among premature male infants and usually resolves spontaneously by descending through the empty scrotum during the first year of the life. Testicular atrophy, infertility, exposure to traumatic events, and malignant degeneration proceed in association with permanent testicular tissue degeneration after 3 years of age if no treatment schedule for the UDT [1-3]. UDT is usually isolated, although it is also seen in some multisystem syndromes including Prader-Willi and EagleBarret. Our patient had no sign for the mentioned syndromes. Unilateral UDT would not impact fertility while bilateral type could loss paternity in $50 \%$ of males [1]. Surgical orchidopexy is the treatment of choice and even in case of testicular atrophy should be considered. Having perfect surveillance approach indicates the latter $[1,2,8]$. GCTs including of seminoma and non-seminoma are the most prevalent type of all testicular cancers. These tumors manifests with pain and mass formation and if metastasize weight loss, back pain, respiratory symptoms, and gynecomastia should be attend. The study patient had not any aforementioned symptoms. Elevated plasma level of tumor markers including of alpha fetoprotein, lactate dehydrogenase, and quantitative beta human chorionic gonadotropin help the diagnosis of GCTs [1]. Patient with intra-abdominal untreated UDT is also susceptible for testicular torsion followed by mass enlargement which is accompanied by acute sever pain, nausea, vomiting, constipation, hematuria, atypical abdominal pain and even shock $[9,10]$. While these signs present it is more familiar with mind to suspect acute appendicitis or diverticulitis in compare with intra-abdominal tortuous UDT [3]. On the contrary, acute appendicitis could present itself with whether unilateral or bilateral scrotal pain, inflammation, mass, abscess formation, and epididymitis [2,8,11-14]. Presence of typical disease history and physical examination for acute appendicitis in a male patient restricts the diagnosis and convince the surgeon to program for emergent classic appendectomy. Although in elderly patient with non-typical clinical findings for diagnosis of acute appendicitis additional imaging study preferably abdominopelvic computed tomographic study with oral and intravenous contras could be helpful. Our patient's either disease history or findings of physical examination were fully in lined with the development of acute appendicitis. Therefore any other additional imaging study was not requested and patient was directly prepared for operating room.

We found just one identical case in the literature which was reported in 1986 by Sarma et al. The mentioned patient was a 48 years old man who sought medical care for his recent abdominal pain. His clinical finding was consistent with the diagnosis of acute appendicitis. Surgical abdominal exploration followed by pathologic study finally implied on invasive UDT seminoma to appendix base that led to acute appendicitis [5]. In the literature, our patient is the second identical case who had typical condition for diagnosis of acute appendicitis while UDT with seminoma component was the underlying pathology.

Regarding the etiology of appendicitis, whenever fecal stasis, fecalith, fruit seeds, lymphoid hyperplasia, parasites, or neoplasm 
block the orifice of the appendix, intraluminal pressure increases following secretion accumulation. The latter then complicated by bacterial colonization which leads to inflammation and development of acute appendicitis [2]. Considering etiology of acute appendicitis lymphoid hyperplasia following to adjacent inflammatory response to presence of the tumor but not obstructive change due to direct tumor invasion, this report was the first and would be remained unique until the next similar one will introduced.

\section{Conclusion}

It was the second case in the literature with Intra-abdominal neglected right UDT presenting itself with acute appendicitis. Seminoma as the most common type of testicular tumor was also confirmed in patient who finally underwent emergent appendectomy. Lymphoid hyperplasia following to adjacent inflammatory response to presence of tumor was terminated to acute appendicitis without direct invasion. Regarding the latter it was the first report in medical literature.

\section{Conflicts of Interest}

We declare that have no conflicts of interest for doing the study.

\section{Acknowledgement}

We present our great acknowledgement to department of urology and also technicians and nurses of Milad Hospital, Kashan, Iran who contributed in performing the work.

\section{References}

1. Brunicardi FC, Anderson DK, Billiar TR (2019) Schwartz's principle of surgery eleventh edition; Mc Graw Hill. 11(2): 1771-1772.

2. Buzatti KC, Gonçalves MV, Silva RG (2017) Acute appendicitis mimicking acute scrotum: a rare complication of a common abdominal inflammatory disease. Journal of Coloproctology. 38: 65-69.
3. Halidou M, Roua A, Amadou Magagi I, Adamou H, Adakal O, et al. (2019) Acute Abdomen Revealing an Unusual Case of Intra-Abdominal Testicular Torsion. Case reports in urology 5815036.

4. Agha RA, Borrelli MR, Farwana R, Koshy K, Fowler A, et al. (2018) For the SCARE Group. The SCARE 2018 Statement: Updating Consensus Surgical CAse REport (SCARE) Guidelines, International Journal of Surgery 60: 132-136.

5. Sarma DP, Weillbeacher TG, Hatem AA (1986) Seminoma arising in undescended testis clinically presenting as acute appendicitis. J Surg Oncol 31(1): 44-47.

6. Sipani M, Bhat A, Prabhakar G (2020) Transverse Testicular Ectopia: A Report of Five Cases and Review of Literature. J Indian Assoc Pediatr Surg 25(6): 404-407.

7. Middleton AH, Martin JM, Wittmann TA, Schmeling GJ (2019) Testicular Dislocation After Pelvic Ring Injury: A Report of 2 Cases. JBJS Case Connect 9(4): e0141.

8. Satchithananda K, Beese RC, Sidhu PS (2000) Acute appendicitis presenting with a testicular mass: ultrasound appearances. Br J Radiol 73(871): 780-782.

9. Staniuk T, Szymański L, Trocha M (2012) Torsed intraabdominal testicular tumor diagnosed during surgery performed due to suspicion of acute appendicitis. Polski Przegląd Chirurgiczny 84(10): 526-530.

10. Twiss C, Grasso M (2000) Abdominal pain associated with an intraabdominal gonad in an adult. Reviews in Urology 2(3): 178-181.

11. Shehzad KN, Riaz AA (2011) Unusual cause of a painful right testicle in a 16-year-old man: a case report. J Med Case Rep 5: 27.

12. Najafizadeh Sari S, Mehdizadeh H, Bagheri Baghdasht MS, Manoochehry S (2017) Suppurative appendicitis presenting acute scrotal pain: a rare condition may confuse surgeons. J Surg Case Rep rjx215.

13. Ozbey I, Aksoy Y, Polat O, Gündoğdu C, Biçgi O (2001) Metachronous tumors of testis in undescendent and ectopic testis. Int Urol Nephrol 33(3): 525-528.

14. Oruc Z, Ebinç S, Kaplan MA (2020) Rare Tumours of the Testis: Twelve Years of Experience. Prague Med Rep 121(3): 181-193. 